\title{
Elasticity Measurement of Soft Tissues using Hybrid Tactile and MARG Based Displacement Sensor Systems
}

\author{
Rory Hampson, Gordon Dobie, and Graeme West
}

\begin{abstract}
Objective: This paper presents a novel soft tissue elasticity measurement technique based on the fusion of Magnetic, Angular Rate, and Gravity (MARG) sensors and fixe d tactile sensors. This work is intended both as a stand-alone technology, and as an extension of traditional tactile imaging of the breast to allow for accurate diagnosis of breast lesions. Methods: A series of artificial silicone materials known to imitate soft biological breast tissues are characterised using the proposed system and compared against an Instron ${ }^{\circledR}$ universal testing machine to determine system accuracy and repeatability. Results: Comparing the characteristics of 10 distinct materials, with elasticities in the range $9 \mathrm{kPa}$ to $90 \mathrm{kPa}$, determined by the proposed system to those from the Instron ${ }^{\circledR}$ yields accuracy within $4 \%$ over the full-scale range. Interexperimental repeatability is within $1.5 \%$. Conclusion: The proposed system delivers absolute elasticity of mate rials to within $4 \%$ which, when combined with its lack of moving parts and low implementation cost, can significantly improve the diagnostic capability of tactile imaging in the clinical environment. Significance: By applying this technique, to determine the background elasticity of breast tissue, in conjunction with the relative lesion elasticity result from tactile arrays, the full non-invasive diagnostic potential of tactile imaging can be realised with the effect of reducing benign biops $y$ rates, secondary care costs, and patient stress.
\end{abstract}

Index Terms-Accelerometers, , Force Measurement, Medical Diagnostic Imaging, Position Control, Tactile Sensors.

\section{INTRODUCTION}

$\mathrm{T}$ HE link between the elasticity, or stiffness, of a breast lesion is well known to be correlated to histological diagnosis [1] [2] [3]. The most common method of detecting breast lesions is the clinical breast exam (CBE) but, as people are unable to quantify the elasticity of a suspected lesion, this normally requires a secondary care referral for a mammogram. The result of the mammogram, an unpleasant and expensive procedure, determines whether surgical biopsy should be

This paper was accepted for publication in IEEE Sensors on 07/07/2019. This work was supported in part by the EPSRC, under funding for $\mathrm{PhD}$ studentships, and by Pressure Profile Systems (PPS) Inc, California, USA. The authors gratefully acknowledge the support of PPS Inc. in the supply of SingleTact ${ }^{\mathrm{TM}}$ Pressure Transducers.

Rory Hampson is with the Centre for Ultrasonic Engineering (CUE), Department of Electronic and Electrical Engineering, University of Strathclyde, 204 George St., Glasgow, G1 1XW, GB (correspondence e-mail: rory.hampson@strath.ac.uk). Gordon Dobie is with the Centre for Ultrasonic Engineering (CUE), University of Strathclyde, GB. Graeme West is with the Advanced Nuclear Research Centre (ANRC), University of Strathclyde, GB. carried out. With over 1 million biopsies performed each year in the United States costing on average between $\$ 290$ and $\$ 380$ each [4], and $80 \%$ resulting in benign diagnosis [5] [6], it is clear that this presents an unacceptable and unnecessary burden on the secondary care system.

Recent developments in industry and academia have focussed on reducing the burden on the secondary care system by allowing for a basic malignant/benign differentiation at home or primary care centres [7]. These developments, based on tactile imaging [8], showed that a $23 \%$ reduction in benign biopsy rate was possible with no missed cancers. Current implementations of tactile imaging use a differential measurement system to determine the relative elasticity of a lesion with respect to the background breast tissue [9], which may be sufficient for a general binary lesion differentiation but for a detailed diagnosis, using any classification algorithm, the absolute elasticity of a lesion, amongst other parameters, is required [10] [11] [12].

\section{A. Hand Held Elasticity Measurement}

Given that absolute elasticity measurement, and material characterisation, can be generalised to a force variation with respect to a compression, or tension, displacement variation, the conventional methods of applying and measuring displacement are applied to portable handheld systems in the literature and in industry.

Optical and hyperspectral camera based techniques have been developed [13], measuring transverse stretching of a lesion for an applied load but, as the camera presents clinical privacy issues this method may face pushback from clinicians and patients when moved from academia to industry.

More traditional methods of measuring elasticity using mechanically driven palpating elements [14] show more promise in terms of public acceptance however, the limitation of the $4 \times 4$ mobile tactile element array reduces the available spatial resolution compared with the fixed element implementation [8] with a $12 \times 16$ fixed element array in a similar area. This means that though an absolute elasticity value is obtained, size and shape information is lost which are each important diagnostic metrics [15].

With these issues in mind, this paper proposes a method of measuring tissue compression, without cameras or palpation guide rails, that can be applied to fixed element tactile arrays, characterising elasticity whilst maintaining the spatial resolution they enjoy. To that end, this paper presents a novel 
sensor displacement measurement technique based on the double integration of compression acceleration, utilising Magnetic, Angular Rate and Gravity (MARG) sensors.

\section{B. MARG Sensor Displacement Measurement}

The measurement of displacement using MARG sensors has been attempted by several eminent authors to varying degrees of success. The primary concern addressed in the literature is the mitigation of the quadratic drift typical of double integration, required to determine displacement from acceleration. The second is the accurate estimation of sensor orientation, required to separate the motion acceleration vector from the static freefall acceleration (gravity) vector.

Rahni and Yahya [16] showed that the orientation estimate obtained using a simple 6DOF integrated accelerometer and gyroscope, or inertial measurement unit (IMU), was insufficient to reliably measure displacement. This was due to the limitations in response time and noise rejection from early complementary filters and Kalman filters, compounded with the reliance on a drifting gyroscope alone to measure orientation. From this point onward a 9DOF MARG is typically used for displacement measurements, as the orientation estimate has been repeatedly shown to be drift free and resilient to motion noise [17][18] [19].

Mitigation of the quadratic drift from double integration is ultimately achieved using expert knowledge concerning the type of motion being measured, namely: knowledge on when either velocity or displacement is equal to a known value.

In the field of human gait tracking, knowing that between steps the velocity of the sensor is zero, the integral drift can be determined and corrected for, allowing for reduction in displacement error shown by Yun et al. [20] where an uncorrected displacement error of $33.3 \%$ can be reduced to $0.3 \%$ by applying the velocity constrained drift correction.

Similarly, in the application of Cardiac motion measurement, Krogh et al. [21] shows that by knowing that the motion of the cardiac wall is cyclic, the displacement between cycles is zero and thus the displacement drift can be measured and corrected for. This type of assumption is more difficult to correct for, in terms of accuracy, relative to the velocity drift compensation as the error source is not restricted to the acceleration integral alone [22].

With these examples in mind, this paper will introduce a new 9DOF MARG displacement algorithm tailored for the specific motion type experienced by an indenter insertion. Concisely, this is the situation where, during an insertion cycle, the velocity, when displacement is maximal, is zero. This means that both displacement and velocity-based compensation methods are utilized, and a full description of this situation and the algorithm is hereby presented and applied to elasticity measurement of soft tissues.

\section{ORGANISATION OF PAPER}

Section III introduces the hardware used in this work, including a detailed description of the components, assembly, and critical operating conditions. Section IV goes on to detail how this hardware is fused to measure the characteristic curve of a test material by proposing the MARG displacement algorithm and explaining how force measurement stability can be used to mitigate the expected integral drift. Section V shows how the algorithm and hardware are validated for the purpose of material characterisation. The materials under test are described, in addition to the ground truth reference measurement. Data processing and cleansing criteria are similarly described in this section. Section VI presents the results of the proposed algorithm and hardware applied to material characterisation by quantifying the accuracy and reliability vs. the ground truth measurement, and the results are presented numerically and graphically for added clarity. Section VII discusses the results and presents the intended future direction of the proposed technology beyond simple material characterisation, within the clinical environment and without. Finally, Section VIII summarises the contribution to knowledge presented in this work and defines the authors' strategic direction regarding the proposed technology.

\section{HARDWARE IMPLEMENTATION}

The proposed algorithm is implemented in hardware using a SingleTact ${ }^{\mathrm{TM}} 15 \mathrm{~mm} 45 \mathrm{~N}$ rated capacitive pressure sensor (Pressure Profile Systems, US-CA), with $<1 \%$ repeatability and $<2 \%$ linearity, is coupled between a $15 \mathrm{~mm}$ diameter hemispherical aluminium indenter and a $15 \mathrm{~mm}$ diameter aluminium probe shaft. The sensor is fixed to the indenter tip and shaft using 3M 300LSE double sided tape (3M Company, US-MN). A $38 \mathrm{~mm}$ diameter acrylic guard is used to protect the sensor from excessive load by decoupling the excess load, when depth is greater than $7.5 \mathrm{~mm}$, from the sensor and transferring it directly to the shaft. An ergonomic handle is applied to the end of the probe shaft, in which the MARG sensor and interfacing microcontroller are housed. The MARG sensor used is a BNO055 (Robert Bosch GmbH, DE), connected to an ATMega32U4 microcontroller (Microchip Technology Inc., US-AZ) running at $16 \mathrm{MHz}$. These sensors are commercially available and communicate via $\mathrm{I}^{2} \mathrm{C}$. They each implement proprietary filters to output their respective variables. The constructed device is shown in Fig. 1.

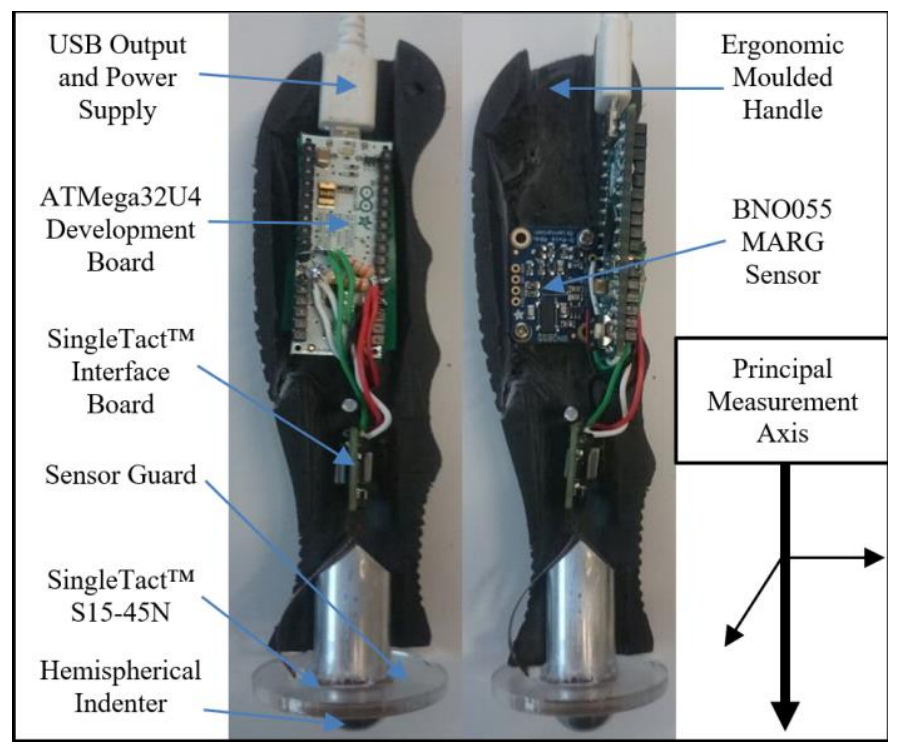

Fig. 1 - Handheld Elastometer hardware design implementation. 
Power is supplied via the Universal Serial Bus (USB) port, also used to interface the measurement system to an external computer running the proposed algorithm. Data is requested from the BNO055 and SingleTact ${ }^{\mathrm{TM}}$ sensors at $100 \mathrm{~Hz}$ and transmitted to the computer immediately without buffering.

The Single Tact ${ }^{\mathrm{TM}}$ interface board converts the capacitance change of the sensor into force and performs internal lowpas $\mathrm{s}$ filtering. The BNO055 MARG sensor is configured to output the absolute orientation quaternion as well as the linear acceleration vector. Its internal fusion algorithm filters the effect of intermittent magnetic fields with the gyroscopes.

Although the handle shape is largely unimportant, what is important is its effect on any elasticity measurement. The elasticity of the handle must be very high with respect to the materials under test such that the elasticity of the handle does not corrupt the elasticity measurement of the material under test. The deformation of the pressure sensor is assumed to be very low, and thus its elasticity very high, with respect to the material under test.

\section{THEORY OF OPERATION}

\section{A. Displacement in the Context of Elasticity}

The device measures the force vs. displacement relationship of an unknown soft material, with the proposed MARG integration algorithm providing the displacement measurement. The displacement of interest, shown in Fig. 2, is the displacement covered by the device from the point of first contact to the point of maximum force, as this will be the point of maximum displacement into the test material during the compression phase. The initial contact velocity, required for this measurement, cannot be determined directly by integration of acceleration, as it is not known when in time the device begins its approach to the material. The proposed algorithm deals with this issue effectively using knowledge that the velocity when the compression period ends is zero. Fig. 2 shows the four principal phases of device operation. The approach period is where the device approaches the test sample linearly, and the compression period contains the displacement required for material characterisation, from the first indenter contact to the point of maximum force.

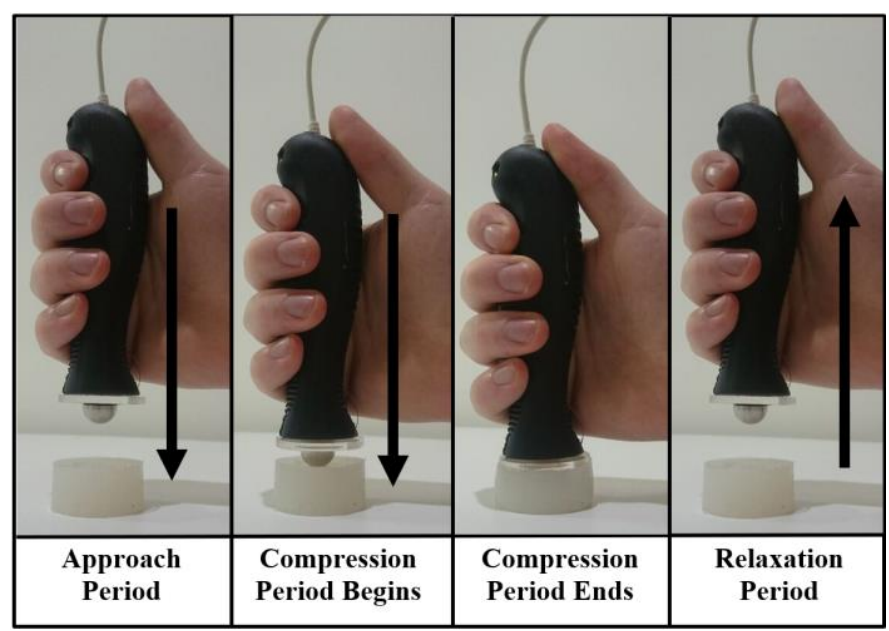

Fig. 2 - Graphical representation of device usage, indicating the direction of compression and the need to apply the device perpendicular to the surface of the test material.

\section{B. Elasticity Specific Algorithm}

The proposed sensor algorithm is shown in Fig. 3. A 9DOF MARG orientation fusion algorithm based on a gradient descent method [19] is implemented using the BNO055. This fuses the magnetic field (B), angular rotation rate $(\dot{Q})$, and the acceleration vector (a), to determine the orientation of the sensor with respect to the gravity vector. This allows for the gravity vector to be separated from the acceleration vector to produce the linear acceleration vector associated with the expected linear motion.

The force $(F)$ measurement is used to trigger and stop the linear acceleration integrator. The force measurement is not subject to the free space vibration noise that the accelerometer is subject to, and so a sharp rise in force is indicative of the start of material compression. Similarly, the turning point of the force measurement indicates material relaxation, and thus triggers the integrator to stop. The accumulated acceleration data between the start and stop periods will then only be associated with the compression of the material.

The force and linear acceleration data for one measurement cycle is shown in Fig. 4, highlighting the meaning of the start/stop conditions. Acceleration magnitude is too small during the relaxation period to reliably measure the material relaxation. The approach period, where the sensors are travelling towards the test material through air, will result in an unknown initial contact velocity.

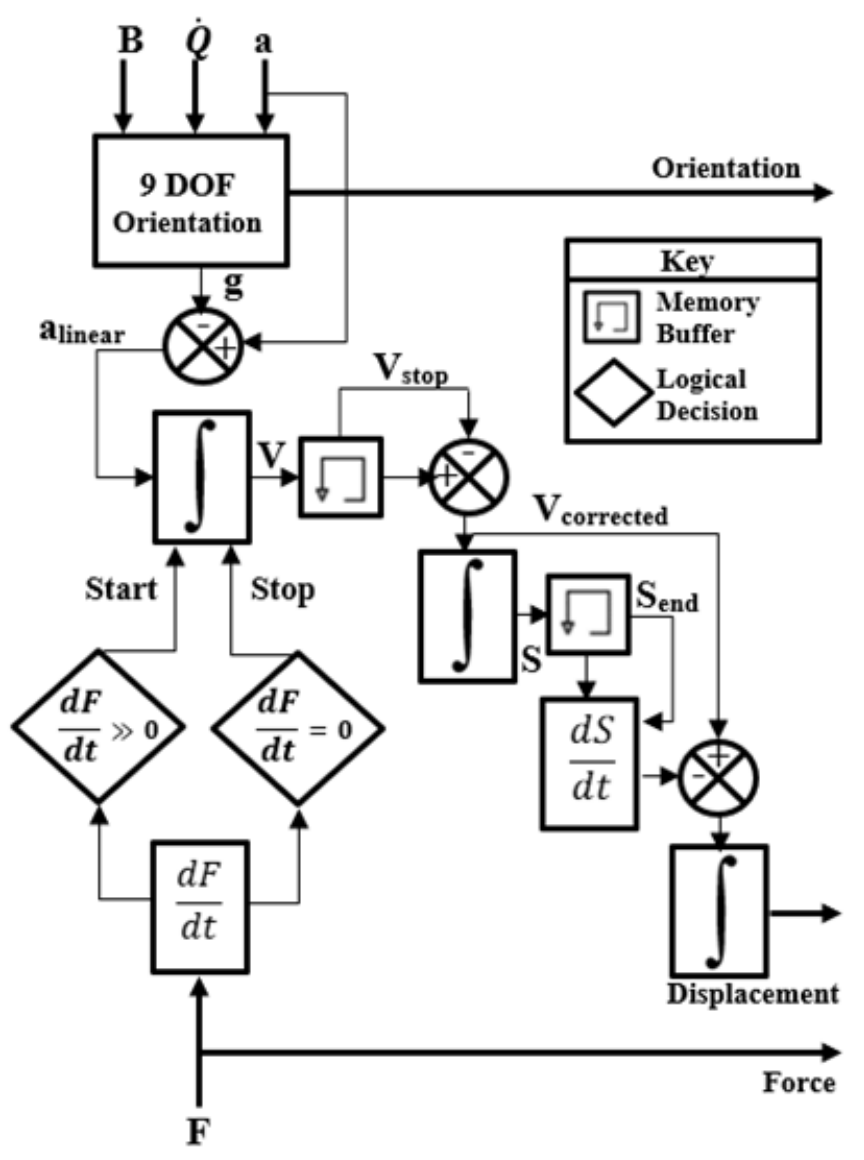

Fig. 3 - MARG sensor algorithm flow diagram. Both velocity and displacement compensation methods are used to estimate the indenter compression depth. Force and displacement are plotted against each other. Orientation is output to assist with valid measurement criteria. 


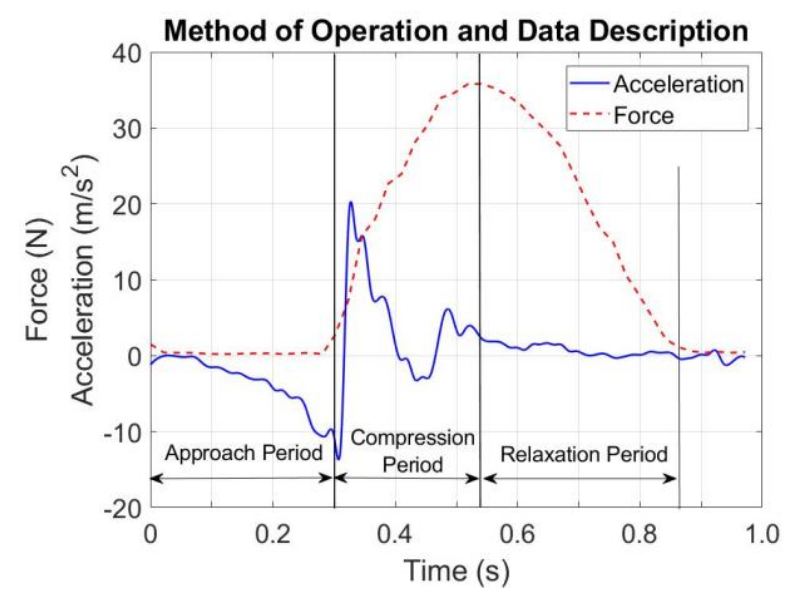

Fig. 4 - Graphical representation of integrator start and stop conditions showing force and acceleration signals for one full measurement cycle. The acceleration on the advancement period will result in the compression beginning with unknown initial velocity.

The acceleration data corresponding to the compression period is integrated to yield velocity $(\mathrm{V})$. The initial velocity parameter is estimated by knowing that the end velocity $\left(\mathrm{V}_{\text {stop }}\right)$ is ideally zero, the estimated velocity can then be offset using this $\mathrm{V}_{\text {stop }}$ value to give the true velocity during the compression cycle ( $\left.\mathrm{V}_{\text {corrected }}\right)$.

The error in $\mathrm{V}_{\text {corrected }}$ is estimated and eliminated by examining the integral displacement (S) with the knowledge that the derivative of displacement, $\mathrm{S}$, must be zero at the end of the compression cycle. Should there be an offset error on $\mathrm{V}_{\text {corrected, }}$ the subsequent displacement gradient after integration, which is $\mathrm{V}_{\text {error }}$, can be removed from $\mathrm{V}_{\text {corrected }}$ and integrated to yield the final displacement estimate (x) that is free from integral drift caused by the estimation of initial velocity.

The displacement vs. time, in the direction of the force sensor principle axis, is then used in conjunction with the force vs. time relationship to determine the material characteristic curve denoted by force vs. displacement. This relationship is then combined with the sensing area and material dimensions to determine the requisite stress vs. strain relationship, characteristic of individual material samples.

The material characteristic curve, the force vs. displacement relationship describing bulk material elastic properties, is generated using a quadratic best fit line to the measured force vs. displacement data from the MARG based device. The quadratic is suitable for representing Hookean and neoHookean materials and provides some resilience to noise.

\section{Hardware Limit Considerations}

As a result of the sensor guard, force measurements at compression depths greater than $7.5 \mathrm{~mm}$ do not follow the expected Hookean or neo-Hookean response as the applied load is no longer being correctly coupled into the sensor and therefore will produce erratic results beyond this point as shown in Fig. 5. Results with depths greater than $7.5 \mathrm{~mm}$ are discounted from the calculation of the quadratic best fit line and estimation material characteristic curve.

The decoupling effect of the sensor guard manifests as a plateau, followed be a sharp spike as the probe compresses the material to the point where the backing dominates the signal.

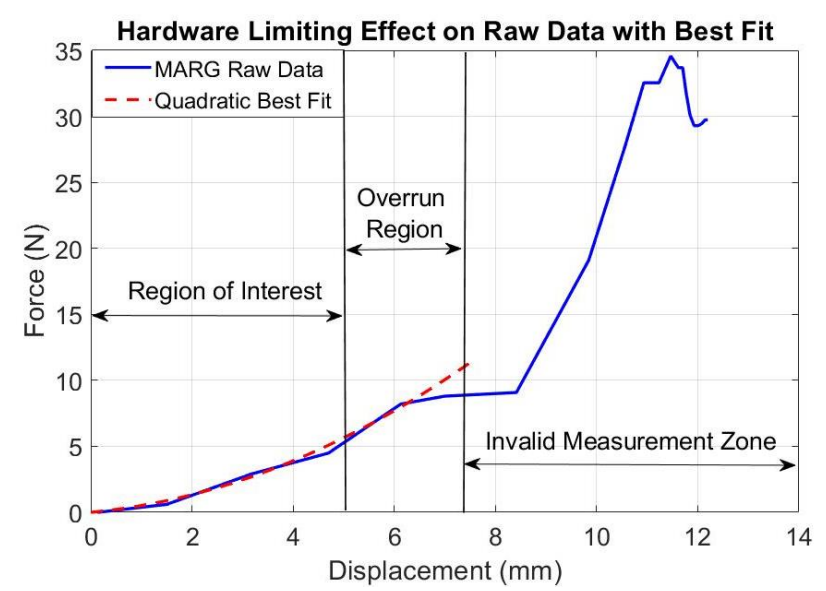

Fig. 5 - Example of the sensor guard invalidating measurements at depths $>7.5 \mathrm{~mm}$. The invalid measurement stems from the change in force vs. displacement direction and rapid change in gradient.

\section{ELASTICITY TEST METHODOLOGY}

\section{A. Elasticity Measurement}

The proposed algorithm and hardware are tested by collecting force vs. displacement measurements using the device, for a range of 10 soft tissue silicone substitutes designed to approximate isolated samples of biological tissues from adipose to fibrous tissue and comparing the mean relationship with that from a universal testing machine.

The device is accelerated linearly, manually, towards the test material, with a contact velocity in the order of $0.8 \mathrm{~m} / \mathrm{s}$ to $1.5 \mathrm{~m} / \mathrm{s}$, depending on the test material. The device is held firmly in the right hand to avoid slippage, and measurements using the device and reference standard are performed in close proximity in both time and space to reduce the effect of material aging and temperature variation. The ambient temperature at the time of testing was $20^{\circ} \mathrm{C}$.

The materials used, the data cleansing required, and a description of the validation methods are now provided.

\section{B. Elasticity Test Materials}

The device is designed to compress the material under test in a linear direction, parallel to the pressure sensor principal axis. The device is pressed into the test material in a fashion akin to a gentle prod, repetitively, in order to be able to generate a range of force vs. displacement solutions whose mean will converge on the true value as the repetitions increase. These force vs. displacement solutions, in themselves are the material stiffness, (k), can then be used to calculate the material elasticity, (E).

Applying the MARG sensor and pressure sensor to elasticity measurements requires soft materials representative of breast tissue to test the system. The test materials used in this trial were made from Ecoflex ${ }^{\mathrm{TM}}, 000-35,00-10$, and 00-30 rated Polydimethylsiloxane (PDMS) two part elastomer (Smooth-on, Inc., US-PA) with differing A:B mixture ratios and additive thinning agents, Silicone Thinner ${ }^{\mathrm{TM}}$ (Smooth-on, Inc., US-PA) to give a range of materials that can approximate human tissues. These materials are listed in Table 1. The silicone elastomer samples were cast into uniform cylindrical billets with a diameter of $38 \mathrm{~mm}$ and a height, $\mathrm{L}$, of $20 \mathrm{~mm}$. 


\section{Valid Measurement Criteria}

Data cleansing is performed in two stages: pre-processing raw data based, and post-processing calculated data based.

Data pre-process cleansing involves immediately rejecting compression data that has a peak acceleration vector component parallel to the principal axis less than $10 \mathrm{~m} / \mathrm{s}^{2}$, approximately freefall. At values below this threshold, quantisation in time and quantisation in acceleration compound to produce unreliable displacement measurements . Additionally, data where the displacement vector direction during compression deviates from the principal axis by more than $5^{\circ}$ is immediately rejected. Beyond this point, the capacitive pressure transducer is experiencing a shear component that it is not designed for. Ideally this constraint would be $0^{\circ}$, but as this is a manually operated device some deviation is inevitable.

Data post-process cleansing removes force, F, vs. displacement, $\mathrm{x}$, best fit curves that do not conform to Hookean or neo-Hookean models that typically describe biological tissues. Such models can be approximated, over a limited range, by a quadratic in the form of (1) as it is known that although biological tissues typically exhibit exponential responses [3], exponentials can be approximated over a limited range by a quadratic [23].

$$
F(x)=a x^{2}+b x-c,\{a b c\} \geq 0
$$

The implications of this is that viscoelastic materials will not be considered, as the test materials can be considered to be neo-Hookean or Hookean. A graphical representation of the data processing measurement rejection conditions is shown in Fig. 6. In this situation, any solution that conforms to (1) is considered in the calculation of the mean response.

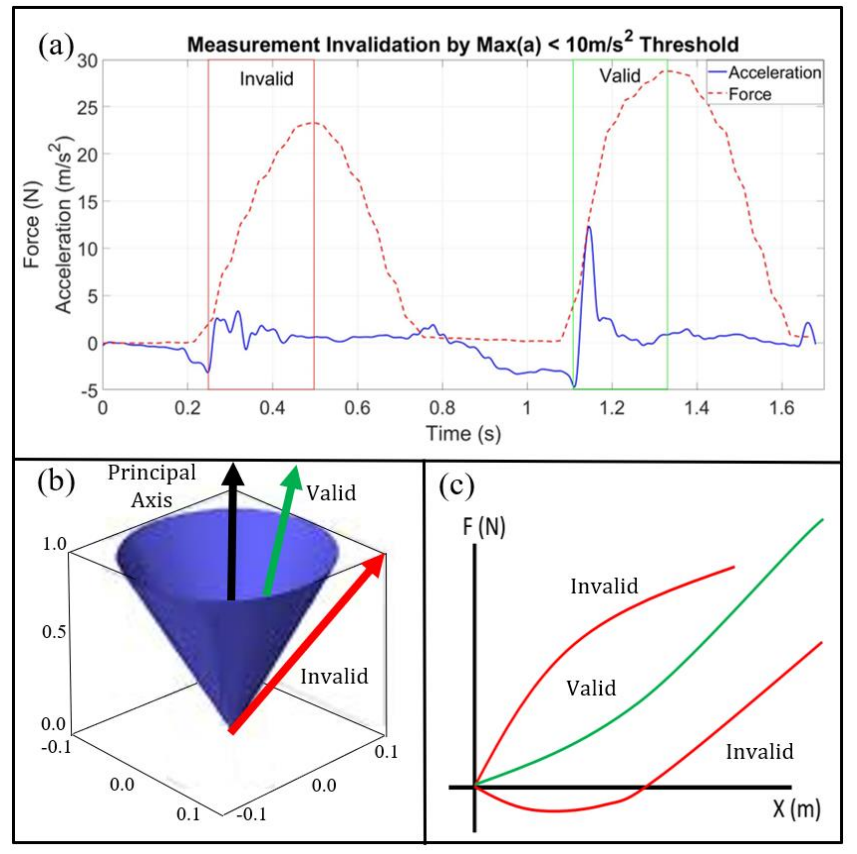

Fig. 6 - Representations of data rejection situations: a) Acceleration magnitude threshold of $10 \mathrm{~m} / \mathrm{s}^{2}$. b) Compression angle deviation threshold of $5^{\circ}$, valid solutions within the cone. $c$ ) Equation form as Hookean or neo-Hookean as in (1).
D. Calculation of Elasticity from a Hemispherical Geometry

For a hemispherical indenter with radius, $r$, the contact area, $\mathrm{A}$, is a nonlinear function of the displacement depth, $\mathrm{x}$, as shown in (2).

$$
A=\pi\left(2 r x-x^{2}\right)
$$

Thus, from the general equation for elasticity (3), the final equation for elasticity using a hemispherical indenter is formed (4), which considers the contact area changes in the estimation of applied stress. In this case the original size of the sample, $\mathrm{L}$, is required and known. In (4), the term $\mathrm{E}(\mathrm{x})$ is used to allow for conditions where $\mathrm{E}$ is not a constant, and is a function of $\mathrm{x}$.

$$
\begin{gathered}
E=\frac{F / A}{\Delta x / L} \\
E(x)=\frac{F(x) / \pi\left(2 r x-x^{2}\right)}{x / L}
\end{gathered}
$$

\section{E. Elasticity Validation Method}

Validation of the calculated characteristic material curve for each material sample is performed by comparing the response from the MARG and pressure sensor system with the response from an Instron ${ }^{\circledR} 3342$ universal testing machine (Instron Engineering Corp., UK). The Instron ${ }^{\circledR} 3342$ has a stated accuracy of $0.5 \%$ and compresses the sample using the same $7.5 \mathrm{~mm}$ hemispherical indenter as the handheld MARG based system. Compression range is restricted to $5 \mathrm{~mm}$, or $25 \%$ strain, rather than the full $7.5 \mathrm{~mm}$, or $37.5 \%$ strain, as at high strains, $\varepsilon>0.33$, thin materials cannot be relied upon to exhibit bulk properties. The pressure sensor is separately tested on each sample material over this range to allow for confidence to be built on each component of this developmental system.

Repeat measurements, for each sample, from the Instron ${ }^{\circledR}$, and the MARG and pressure sensor system are performed, and mean values are used to compare the material characterisation ability of each method. The spread of results, from the repeat measurements, are presented to show the accuracy of any single measurement and compared to show the reliability of the MARG based system vs. the Instron ${ }^{\circledR}$ standard.

\section{RESULTS}

\section{A. MARG Sensor Elasticity Results}

The results of sequential compressions of 10 silicone samples by the MARG based system and the Instron ${ }^{\circledR}$ standard are shown in Table 1. The elasticities for each material are calculated using the linear region of (4) and are logged as the mean response with the interexperimental variability indicated separately. The mean error in the MARG based estimation of elasticity is taken as the total error between the mean elasticity estimations from the Instron ${ }^{\circledR}$ using 4 trials per sample and MARG using 10 trials per sample. These results are presented in graphical form in Fig. 7. 
Table 1 - Material compositions with measured elasticity. Elasticity is taken as the mean of repeated cycles, with spread indicated.

\begin{tabular}{|c|c|c|c|c|c|}
\hline \multicolumn{6}{|c|}{$\begin{array}{c}\text { MATERIAL COMPOSITIONS FOR ELASTICITY } \\
\text { MEASUREMENT TRIAL }\end{array}$} \\
\hline Sample & $\begin{array}{c}\text { Material } \\
\text { A:B } \\
\text { Ratio }\end{array}$ & $\begin{array}{c}\text { Thinner } \\
\text { By } \\
\text { Mass }\end{array}$ & $\begin{array}{c}\text { ERef }_{\text {Ref }} \\
(\mathbf{k P a})\end{array}$ & $\begin{array}{c}\text { EMARG } \\
(\mathbf{k P a})\end{array}$ & $\begin{array}{c}\text { Mean } \\
\text { Error } \\
(\%)\end{array}$ \\
\hline 1 & $\begin{array}{c}000-35 \\
1: 1\end{array}$ & $+10 \%$ & $\begin{array}{c}8.9 \\
+0.1 \\
-0.1\end{array}$ & $\begin{array}{c}\mathbf{9 . 1} \\
+0.9 \\
-0.5 \\
\end{array}$ & 2.24 \\
\hline 2 & $\begin{array}{c}000-35 \\
1: 2\end{array}$ & $+20 \%$ & $\begin{array}{l}\mathbf{1 2 . 3} \\
+0.2 \\
-0.1\end{array}$ & $\begin{array}{l}12.1 \\
+0.6 \\
-0.4\end{array}$ & -1.62 \\
\hline 3 & $\begin{array}{c}00-10 \\
1: 1\end{array}$ & $+10 \%$ & $\begin{array}{r}\mathbf{1 4 . 7} \\
+0.2 \\
-0.1 \\
\end{array}$ & $\begin{array}{r}\mathbf{1 5 . 1} \\
+0.5 \\
-0.2 \\
\end{array}$ & 2.72 \\
\hline 4 & $\begin{array}{c}00-10 \\
1: 2\end{array}$ & $+0 \%$ & $\begin{array}{l}\mathbf{1 5 . 7} \\
+0.5 \\
-0.3\end{array}$ & $\begin{array}{l}15.2 \\
+0.7 \\
-0.3\end{array}$ & -3.18 \\
\hline 5 & $\begin{array}{c}00-10 \\
2: 1\end{array}$ & $+10 \%$ & $\begin{array}{l}\mathbf{2 2 . 1} \\
+0.2 \\
-0.2\end{array}$ & $\begin{array}{l}\mathbf{2 2 . 1} \\
+1.0 \\
-0.5\end{array}$ & -0.11 \\
\hline 6 & $\begin{array}{c}00-10 \\
2: 1\end{array}$ & $+20 \%$ & $\begin{array}{l}29.5 \\
+0.1 \\
-0.3\end{array}$ & $\begin{array}{r}30.1 \\
+1.1 \\
-0.9 \\
\end{array}$ & 2.03 \\
\hline 7 & $\begin{array}{c}00-10 \\
1: 3\end{array}$ & $+0 \%$ & $\begin{array}{l}34.9 \\
+0.3 \\
-0.2\end{array}$ & $\begin{array}{l}\mathbf{3 4 . 7} \\
+1.9 \\
-1.0\end{array}$ & -0.57 \\
\hline 8 & $\begin{array}{c}00-30 \\
1: 1\end{array}$ & $+0 \%$ & $\begin{array}{l}\mathbf{5 5 . 2} \\
+0.2 \\
-0.4\end{array}$ & $\begin{array}{l}\mathbf{5 7 . 3} \\
+1.8 \\
-1.5\end{array}$ & 3.80 \\
\hline 9 & $\begin{array}{c}00-30 \\
2: 1\end{array}$ & $+10 \%$ & $\begin{array}{l}73.8 \\
+0.3 \\
-0.1\end{array}$ & $\begin{array}{l}71.1 \\
+2.5 \\
-1.6\end{array}$ & -3.65 \\
\hline 10 & $\begin{array}{c}00-30 \\
1: 3\end{array}$ & $+0 \%$ & $\begin{array}{r}89.6 \\
+0.2 \\
-0.2 \\
\end{array}$ & $\begin{array}{r}\mathbf{9 2 . 4} \\
+2.9 \\
-2.1\end{array}$ & 3.12 \\
\hline
\end{tabular}

It can be seen from Table 1 that the worst-case error between the mean responses of the MARG based system and the Instron reference is less than $4 \%$ over the material range.

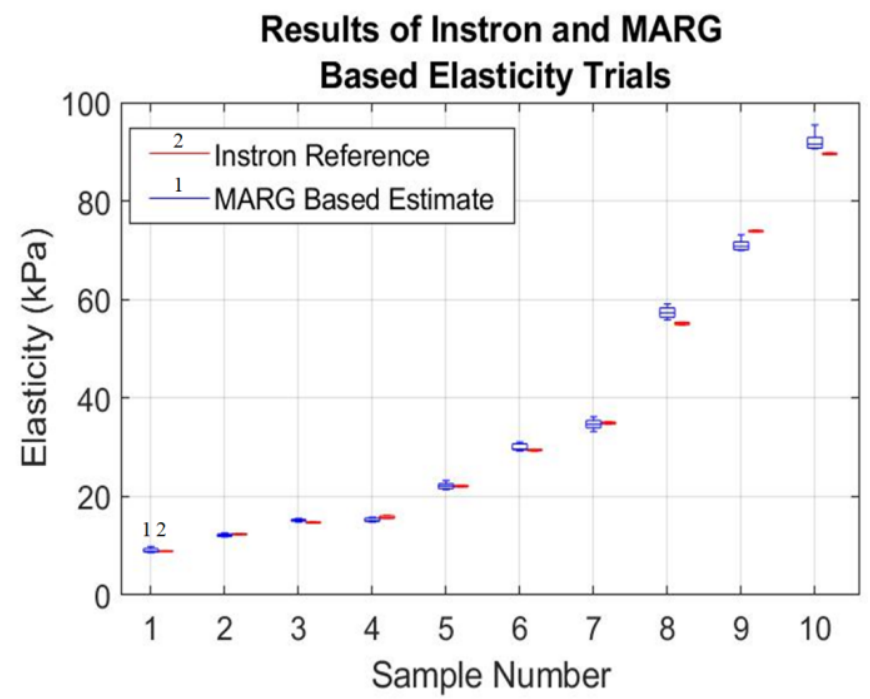

Fig. 7 - Representation of full experimental results, showing the range of elasticities covered, the relative spread of each measurement and the agreement between the MARG system and Instron. The samples have been ranked for clarity.

\section{B. MARG Based System Performance Analysis}

From the results in Table 1, the sensitivity of the MARG based system to errors borne from elasticity related effects of the material under test is shown in Fig. 8. Additionally, the total intraexperimental scatter, for 10 repeated MARG tests per sample, is shown in Fig. 9. These graphs are required to characterise the response of the MARG based system over its operational range.
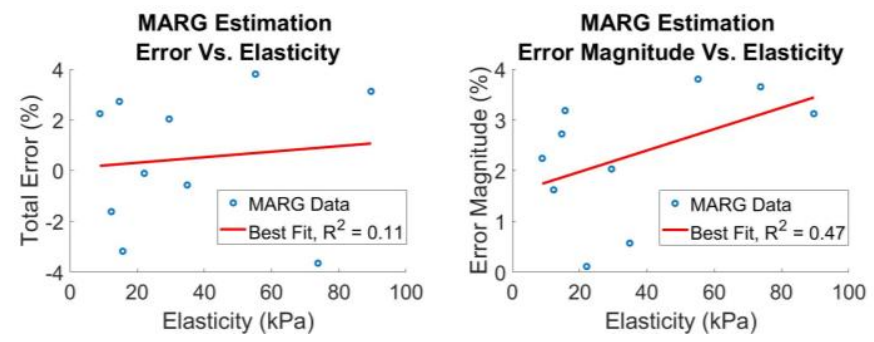

Fig. 8 - Systemic Error Vs. Measured Elasticity, Left) Total Error Vs. Elasticity, showing a very weak correlation. Right) Error Magnitude Vs. Elasticity, showing a stronger correlation, but still weak.
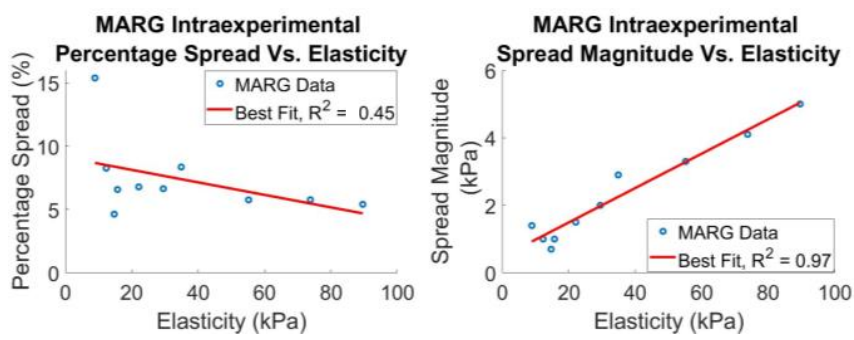

Fig. 9 - Intraexperimental scatter Vs. Elasticity, Left) Spread as a percentage of measured Elasticity showing a weak negative correlation. Right) Spread magnitude, showing a very strong positive correlation.

\section{Comparison of Compression Number Sensitivity} and Inter Test Variability For Sample 3

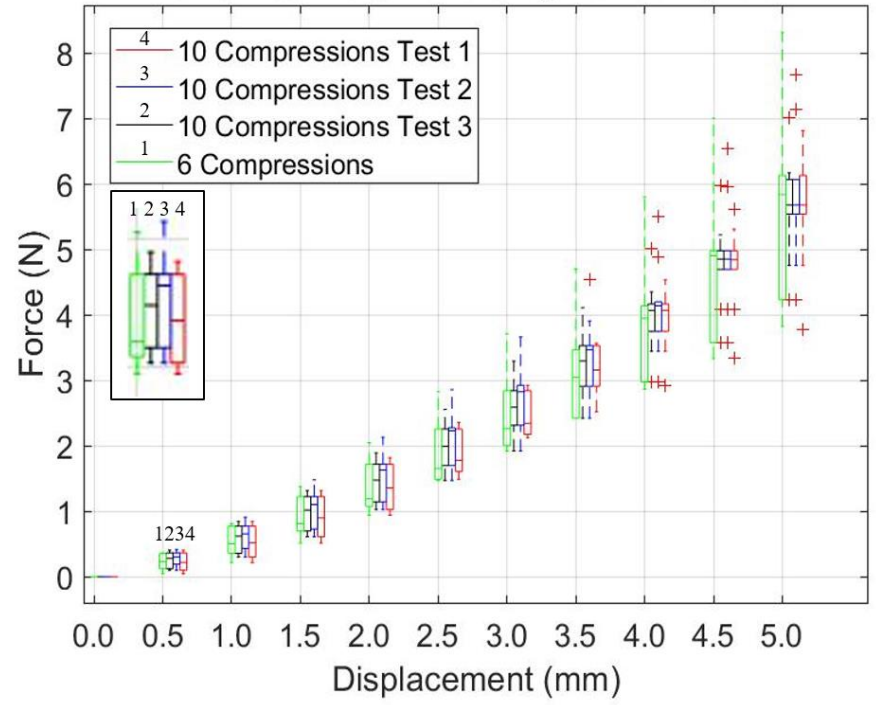

Fig. 10 - Comparison between 6 compressions per test and 10, showing overall scatter decreases with compression number, and multiple sequential tests with constant compression numbers yield repeatable interexperimental results within $1.5 \%$. 
The results of repeatibility testing on sample 3 , chosen at random, is shown in Fig. 10. It can be seen that utilising a 10 compression cycle measurement regime rather than a 6 cycle regime significantly reduces the overall estimation spread. This is expected as statistical outliers are easier to identify with higher population numbers.

Additionally, it is shown that 3 repeated 10 cycle measurements produce excellent repeatability over the full compression range. This provides good confidence in the measurement technique as we are able to achieve repeatability on the raw force vs. displacement response even before statistical outlier rejection is applied. This single sample repeatibility is typical of the global system repeatibility and, when mean responses are considered, results in mean repeatability within $1.5 \%$.

\section{DisCUSSION}

\section{A. Elasticity Results Discussion}

The better than $4 \%$ measurement accuracy of the proposed system, shown in Table 1 and Fig. is achieved by taking the mean response of 10 repeated compression cycles and comparing this with the mean response of the Instron®. This is appropriate, as even the Instron ${ }^{\circledR}$ uses internal averaging, but the Instron ${ }^{\circledR}$ can achieve this in one compression.

If any single compression cycle were taken as being representative of the material under test the accuracy would be considerably worse, up to $50 \%$ as indicated in Fig. 10. Additionally if fewer compression cycles are taken, say 6 for example, outliers cannot be easily identified and so the accuracy of any single compression used to calculate the mean response will also be up to $50 \%$. However by taking 10 cycles, outliers can be identified and rejected from the mean calculation resulting in the error of any single compression used to calculate the mean typically being between $5 \%$ and $10 \%$ as shown in Fig. 9.

If any single full measurement of a tissue, defined as 10 compression cycles, is used to characterise a tissue then the accuracy becomes better than $4 \%$ when compared with the Instron ${ }^{\circledR}$. In order to facilitate a timely measurement, 10 cycles are used for each measurement as this gives acceptable accuracy in a reasonable timeframe $(<10 \mathrm{~s})$.

\section{B. Error Sources and Limitations}

There are 2 primary sources of error in the proposed system. The first is error arising from the measurement of applied stress. One of the key requirements of this technique is that the probe compresses normally to the surface and have a direction vector within $5^{\circ}$ of the sensor axis. This angle is used as an inexperienced operator can be expected to achieve a successful compression $95 \%$ of the time. Ideally this would be $0^{\circ}$, but people cannot move linearly without guidance due to the hinging nature of the elbow. Consequently, if the angle is not $0^{\circ}$ the stress measurement will not correlate with the strain measurement but, as the angle is low, the shear force is low. The parasitic effects of the capacitive sensor in contact with biological tissues is not a significant source of error as the sensors are fully shielded. Fig. 11 shows the effect of touching the sensor on the noise floor of the force measurement. The effect of the temperature coefficient, $<0.2 \% /{ }^{\circ} \mathrm{C}$, is negligible.
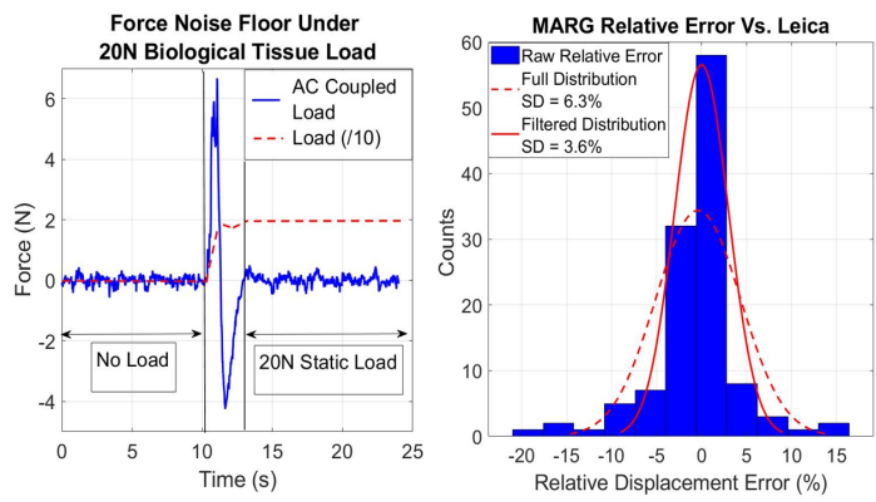

Fig. 11 - Error and repeatability plots, Left) Force sensor noise does not noticeably increase near biological tissues, so is immune to parasitic effects. Right) Comparison of MARG displacement Vs. Leica Tracker, showing that the overall error is reduced by applying the measurement criteria described in section V.C.

The second is error arising from the measurement of strain. As this is done by the double integration of acceleration, this is certainly the dominant source of error, despite the effect of the MARG fusion algorithm in reducing the effect of integral drift. The measurement of strain is most affected by vibration, as the direction is filtered using magnetometers and gyroscopes but the strain is only determined by the acceleration, as such the measurement of strain is resistant to intermittent magnetic field variation. Fig. 11 shows the validation of the displacement measurement vs. a Leica ${ }^{\circledR}$ AT403 Absolute Tracking system (Hexagon Metrology Ltd. UK) with accuracy $\pm 15 \mu \mathrm{m}$ over 120 manual repetitions on sample 3. These were at a range of depths between $8 \mathrm{~mm}$ and $15 \mathrm{~mm}$. This shows the effect of the valid measurement criteria on error reduction. The increase in elasticity spread magnitude vs. elasticity, shown in Fig. 9, indicates that the vibration gets worse as the materials stiffen. This is expected as the probe compresses the material harder, the stability of the hand reduces and so shakes the sensor. This results in variation in the strain measurement by constructive or destructive addition of the acceleration vectors.

\section{Operational Range Compared with Typical Breast Tissues}

As the proposed system is capable of measuring elasticity in the range $9 \mathrm{kPa}$ to $90 \mathrm{kPa}$ with accuracy within $4 \%$, up to $115 \mathrm{kPa}$ based on the tactile sensor rating, it is possible to characterise the elasticity of a broad range of breast tissues directly including several malignant conditions such as Phyllodes Tumours and Papilloma [1] [3]. Given that the tactile sensors can by operated up to 2 times their rating with proper calibration, the range increases to $250 \mathrm{kPa}$. This allows for measurements of ex-vivo samples of malignant tissues such as Lobular Carcinoma and Fibroadenoma at strains $<0.1$.

The intended future application of this system is as a support method for larger tactile imaging systems, where this method would be used to measure the background breast elasticity, allowing for characterisation of in-vivo lesions by differential measurement [8]. Consequently the system would be typically used on normal tissue and soft benign tissues [23], with elasticities of approximately $10 \mathrm{kPa}$ and $88 \mathrm{kPa}$ for fatty tissue and glandular tissues respectively. This is well within the characterised operational range of the proposed system. 


\section{Applications and Commercial Implications}

The application of this technology is in fixed array tactile imaging, allowing for breast and lesion elasticity to be reliably determined together in a non-invasive manner, where this technology provides the background breast elasticity. This is an important step towards tactile imaging performing on the spot diagnosis of lesions, as commercial tactile imaging provides only the relative elasticity of a lesion currently.

Additionally, the development of an elastometer using no moving parts presents an important commercial implication. The lack of complex mechanisms will significantly reduce the implementation cost and improve the mean time between failures (MTBF) when compared to currently available mobile element methods.

\section{CONCLUSIONS}

This paper has presented a novel, hand-held, elasticity measurement technique based on strain measurements from the double integration of acceleration from MARG sensors, capable of characterising soft tissues with elasticities in the range of $9 \mathrm{kPa}$ to $90 \mathrm{kPa}$, with a maximum effective range of $115 \mathrm{kPa}$. The accuracy when compared with an Instron ${ }^{\circledR}$ Universal Testing Machine is within 4\%, with a repeatability of $1.5 \%$ which can be fully explained by material variability.

To achieve this result, a novel MARG fusion algorithm was also presented. The algorithm fused the tactile sensor and MARG sensor, using the knowledge that the MARG estimated strain should follow the same trend as the measured stress. This algorithm allows for elimination of the quadratic drift in displacement, typical of double integration of acceleration.

The clinical significance of measuring elasticity of breast tissues is clear in that the material elasticity is correlated with histological diagnosis and identification of a tissue. The operating range of the proposed system, covers the elasticity ranges of fatty tissue, glandular tissue, and several malignant tumours and carcinomas. This means that, although this technique is intended to support and supplement more established tactile imaging methods by measuring background tissue elasticity, which allows for in-vivo characterisation of lesions, the proposed system is fully capable of stand-alone usage as an elastometer for pathologists in the clinical environment, although this would be in ex-vivo conditions.

The application of this technique to general fixed array tactile imaging systems will provide additional diagnostic metrics, enabling detailed lesion diagnosis in the near future at primary care centres or at home. This will have the positive effect of reducing the burden and cost of unnecessary mammograms and biopsies on secondary care centres and fundamentally reducing patient stress.

\section{REFERENCES}

[1] P. Wellman, "Tactile Imaging - PhD Thes is," University of Harvard, Harvard, 1999.

[2] T. A. Krouskop, et al., "Elastic moduli of breast and prostate tissues under compression," Ultrasonic Imaging, vol. 20, pp. 260-274, 1998

[3] P. Wellman, R. H. Howe, E. Dalton and K. A. Kern, "Breast tissue stiffness in compression is correlated to his tological diagnosis," Harvard Biorobotics Laboratory, Technical Report, 1999.
[4] University of Pennsylvania School of Medicine, "Including diagnos is related costs, 3-D mammography costs less than digital mammography," ScienceDaily, 8 December 2017. [Online]. Available: https ://www.sciencedaily.com/releases/2017/12/171208085323.htm. [Accessed 18 February 2019].

[5] W. Liang, W. Lawrence and C. B. Burnett, "Acceptability of diagnostic tests for breast cancer,” Breast CancerRes. Treat., vol. 79, pp. 199-206, 2003 .

[6] D. Gur, L. P. Wallace and A. H. Klym, "Trends in recall, biopsy, and positive biopsy rates for scrrening in mammography in an academic practice," Radiology, vol. 235, pp. 396-401, 2005.

[7] V. Egorov, et al., "Differentiation of benign and malignant breast lesions by mechanical imaging," Breast Cancer Res. Treat., vol. 118, pp.67-80, 2009.

[8] V. Egorov and A. P. Sarvazyan, "Mechanical Imaging of the Breast," IEEE TMI, vol. 27, no. 9, pp. 1275-1287, 2008.

[9] A. P. Sarvazyan and V. Egorov, "Mechanical Imaging in Medical Applications," in IEEE EMBS, Minneapolis, 2009.

[10] B. Zheng, S. W. Yoon and S. S. Lam, "Breast cancer diagnosis based on feature extraction using a hybrid of k-means and support vector machine algorithms," Expert Systems with Applications, vol. 41, no. 4, pp. 14761482, 2014.

[11] A. Bhardwaj and A. Tiwari, "Breast cancer diagnos is using Genetically Optimized Neural Network model," Expert Systems with Applications, vol. 42, no. 10, pp. 4611-4620, 2015.

[12] R. W. Woods, G. S. Sisney, L. R. Salkowski, K. Shink, Y. Lin and E. S. Burnside, "The mammographic density of a mass is a significant predictor of breast cancer," Radiology, vol. 258, no. 2, pp. 417-425, 2011.

[13] A. Sahu, et al., "Characterization of Mammary Tumors Using Noninvasive Tactile and Hyperspectral Sensors," IEEE Sensors, vol. 14, no. 10, pp. 3337-3344, 2014.

[14] R. B. Broach, et al., "A cost-effective handheld breast scanner for use in low-resource environments: a validation study," World Journal of Surgical Oncology, vol. 14, no. 1, 2016.

[15] R. M. Rangayyan, et al., "Measures of Acutance and Shape for classification of breast tumors," IEEE Transactions on Medical Imaging, vol. 16, no. 6, pp. 799-810, 1997.

[16] A. A. A. Rahni and I. Yahya, "Obtaining Translation from a 6-DOF MEMS IMU - an overview,” in APACE, Melaka, 2007.

[17] R. G. Valenti, I. Dryanovski and J. Xiao, "Keeping a Good Atttitude:A Quaternion-Based Orientation Filter for IMUs and MARGs," Sensors, vol. 15, pp. 19302-19330, 2015.

[18] B. Fan, Q. Li, C. Wang and T. Liu, "An Adapthive Orientation Estimation Method for MAgnetic and Inertial Sensors in the Presence of Magnetic Disturbances,"Sensors, vol. 17, no. 6, p. 1161, 2017.

[19] S. O. H. Madgwick, A. J. Harris on and R. Vaidyanathan, "Estimation of IMU and MARG orientation using a gradient descent algorithm," in ICORR, Zurich, 2011

[20] X. Yun, et al., "Self-contained position tracking of human movement using small inertial/magnetic sensormodules," in IEEE International Conference on Robotics and Automation, Rome, 2007.

[21] M. R. Krogh, et al. "Gravity Compensation Method for Combined Accelerometer and Gyro Sensors Used in Cardiac Motion Measurements," Annals of Biomedical Engineering, vol. 45, no. 5, pp. 1292-1304, 2017.

[22] P. Neto, J. N. Pires and A. P. Moriera, "3-D position estimation from inertial sensing:minimising the error from the process of double integration of accelerations," in IECON, Vienna, 2013.

[23] Massachusetts Institute ofTechnology, "18.01SC Single Variable Calculus - Quadratic Approximation at 0 for Several Examples," MIT, Mass achusetts, 2010.

[24] M. Guray and A. A. Sahin, "Benign Breast Diseases: Classifiacation, Diagnosis, and Management," The Oncologist: Breast Cancer, vol. 11, pp. 435-449, 2006. 
Rory Hampson received the Meng degree from the University of Strathclyde, Glasgow, U.K., in 2017. He is currently a $\mathrm{PhD}$ researcher in the Department of Electronic and Electrical Engineering at the University of Strathclyde. His current work focusses on tactile imaging for breast cancer diagnosis, and medical decision making based on tactile sensor technology. His research interests include Medical Imaging, Inertial Guidance and Control, Mechanical Modelling, and Engineering for Sustainable Development.

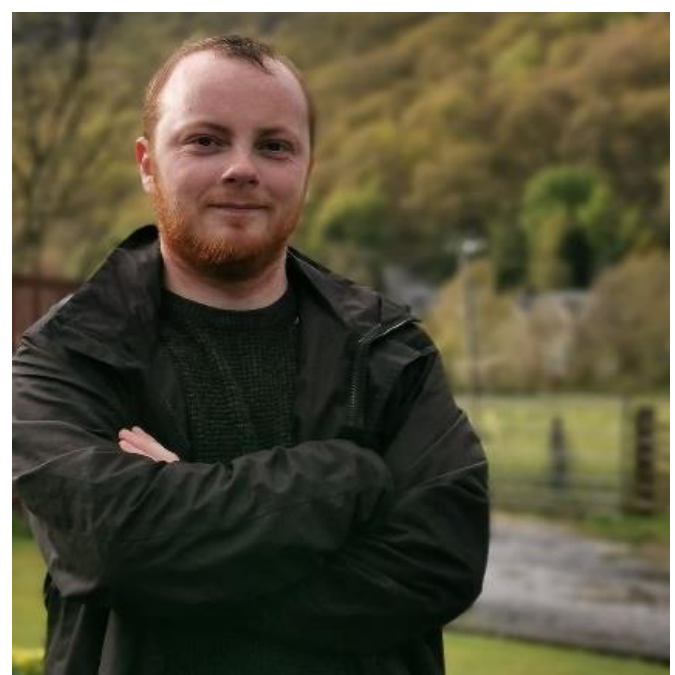

Dr Gordon Dobie is a Senior Lecturer in Electronic \& Electrical Engineering at the University of Strathclyde, UK. He has 12 years' experience and almost 60 publications in automation, structural inspection, sensors and signal processing. Dr Dobie has a strong track record in industrial engagement working with partners in the Oil and Gas, Nuclear and Healthcare sectors to deliver novel solutions to real industrial problems. His work led directly to an onsite robotic inspection of a critical path reprocessing vessel at Sellafield. Dr Dobie has licensed key parts of his team's mobile robotics technology to SME members of the Research Centre for Non-Destructive Evaluation. He currently leads a team of $12 \mathrm{PhD} / \mathrm{EngD}$ students and postdoctoral researchers.
Dr. Graeme West received the B.Eng. (Hons.) and Ph.D. degrees from the University of Strathclyde, Glasgow, U.K., in 1998 and 2002, respectively. He is a Senior Lecturer in the Department of Electronic and Electrical Engineering at the University of Strathclyde. His research interests include intelligent system applications in condition monitoring, diagnostics and prognostics, particularly applied to the nuclear power generation industry.

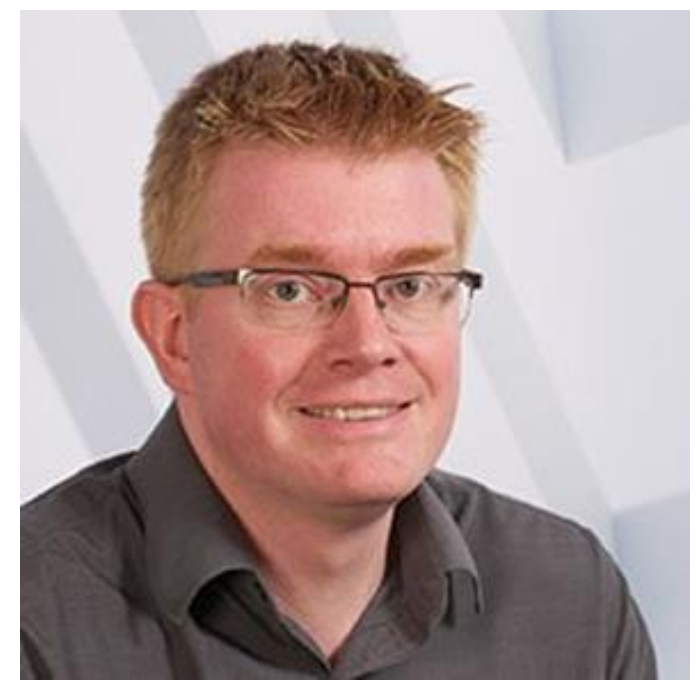

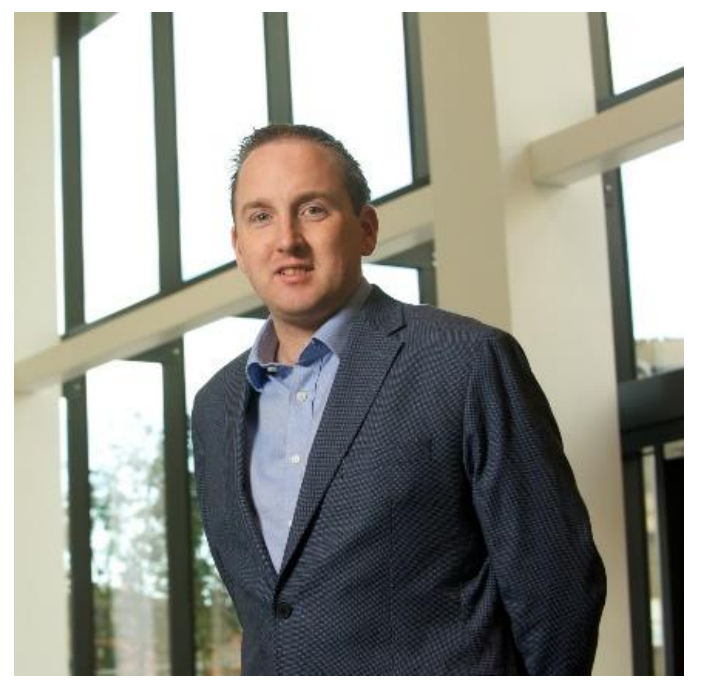

